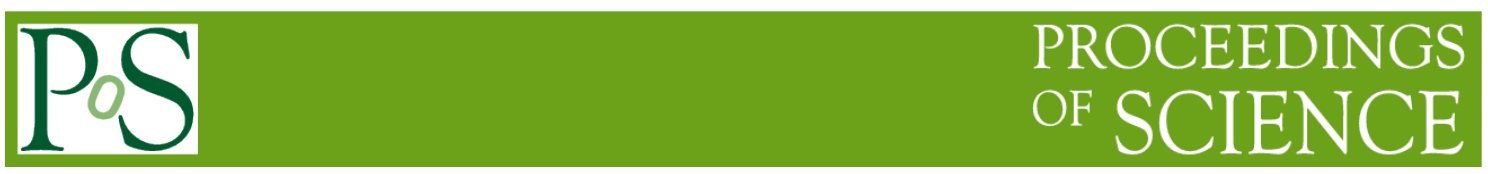

\title{
A Novel Plan for Crime Scene Reconstruction
}

Guangjun Liao ${ }^{123}$

Faculty of Forensic Science and Technology, Guangdong Police College

Guangzhou, China 510320

E-mail: 56114827@qq.com

\section{Yali Zheng}

School of Automation Engineering, University of Electronic Science and Technology of China

Chengdu, China 611731

E-mail:8272335@qq. com

\section{Lixuan Zhao, Xuedong Wu}

Faculty of Forensic Science and Technology, Guangdong Police College

Guangzhou, China 510320

\begin{abstract}
The traditional methods of registering crime scene, such as photographs, sketches, videos and oral descriptions, etc., are lowly efficient and couldn't meet the requirement. In this paper, a new method for crime scene reconstruction which combines the virtual reality techniques with efficient interaction is hereby proposed. The new techniques, such as close-range photogrammetry, panorama photography and crime scene virtual tour, are used for recoding and showing the complex crime scene. The case study shows it's a good way for analyzing crime scene.
\end{abstract}

CENet2015

12-13 September 2015

Shanghai, China

\section{${ }^{1}$ Speaker}

${ }^{2}$ Correspongding Author

${ }^{3}$ The research was sponsored by the Applied Creation Research Program of Ministry of Public Security (Project No.2012YYCXGDST081), Youth Fund Project of Education of Guangdong for Outstanding Young Teachers in University and Program for Innovative Research Team of Guangdong Police College (2015JSTD03). 


\section{Introduction}

Crime scene is the location where the accident occurs, leaving the most physical evidence [1]. It is necessary to register the detailed information by precise protocols and using measurement techniques.

The description for crime scene should be objective, accurate, complete, noninvasive and digitally storable for subsequent analysis and reconstruction; however, the traditional methods of registering crime scene, such as photographs, sketches, videos and oral descriptions, etc., aren't effective and objective.

For the virtual crime scene reconstruction, recording and displaying the scene is crucial [2]. In recent years, 3D laser scanning is a promising method, which allows investigators to return the site to retrieve the missing or forgotten details [3]. On the other hand, virtual reconstruction was applied in the field, such as SL Davy-Jow, et al [4] presented a novel application of anthropometry in a child death, T ColardColard T, e] introduced a method of 3D crime scene reconstruction for bullet trajectory. MH MaMinhua Ma, e described 3D animation and virtual reality in forensic visualization. In general, the reconstruction generally involves a few of steps, which are shown as Fig.1.

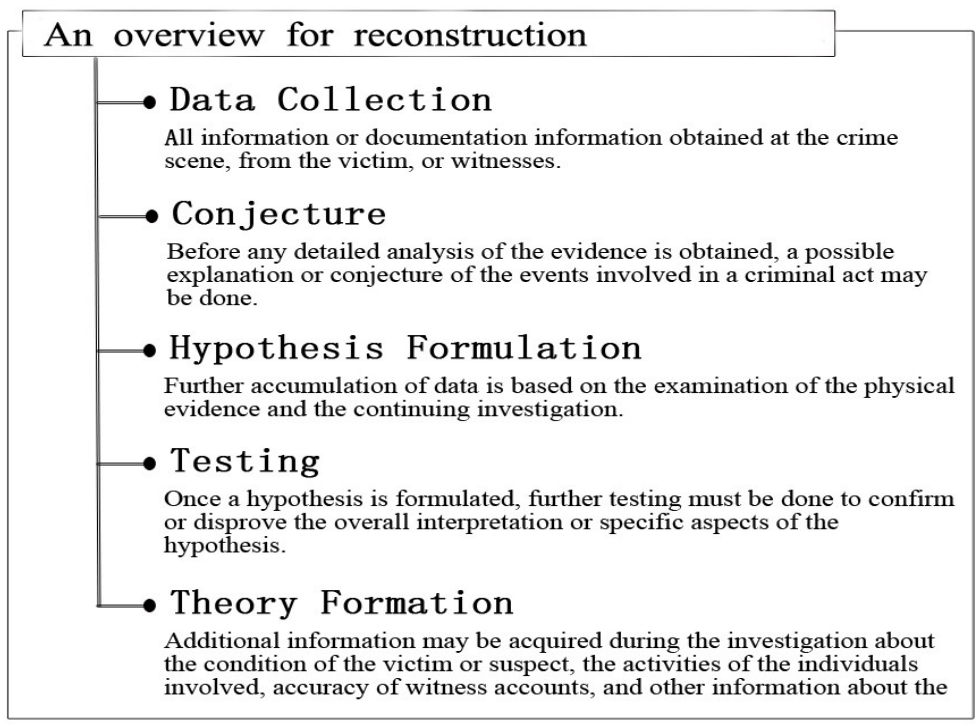

Figure 1: an overview for reconstruction

Nevertheless, there are few plans for the crime scene virtual reconstruction. In order to solve this problem, we combine close-range photogrammetry, virtual reality, and panorama, crime process simulation based on 3D modeling software like 3D-maxs and poser, etc. .

\section{Methodology}

\subsection{Recording 3D Information by Close-range Photogrammetry}

Compared with the laser scanners, the close-range photogrammetry is a more accurate and viable measurement tool, which consists of a consumer-grade digital camera and a photogrammetry software program. It has been used in many diverse applications in the field, such as accident reconstruction and industry, etc. [5-7]. The close-range photogrammetry using consumer-grade digital camera typically consists of five steps:

(1) The consumer-grade digital camera calibration through the special procedure;

(2) Record at least two images;

(3) Mark the corresponding points in two or more images;

(4) Establish the relationship between the image coordinate and the world coordinate; 
(5) Produce the XYZ coordinate by inputting the image coordinates.

\subsection{Panorama of Crime Scene}

Panorama is a view or series of pictures that shows all the features of a subject. In order to rebuild the crime scene's panorama, there are mainly two methods. One method is based on IBR (Image Based Rendering), which is associated with virtual reality and image processing [8]. The other is based on the texture mapping, which uses 3D modeling software such as 3D MAX, Poser and some other software.

\subsubsection{Panorama Based on IBR}

As to the panorama based on IBR, generally, it is recommended for full frame camera to produce quality images [9]. In addition, it is necessary for a suitable software for stitching photographs, correcting white balance, and so on.

\subsubsection{Panorama Based on Texture Mapping}

There are many software packages for 3D modeling and animation ranging from professional applications [10]. In the field of virtual crime reconstruction, compared with the panorama based on IBR, it could use the data acquiring close-range photogrammetry rationally, and it could simulate different conditions of the crime scene. Fig.2 shows the process of reconstruction the crime scene based on 3D modeling software.

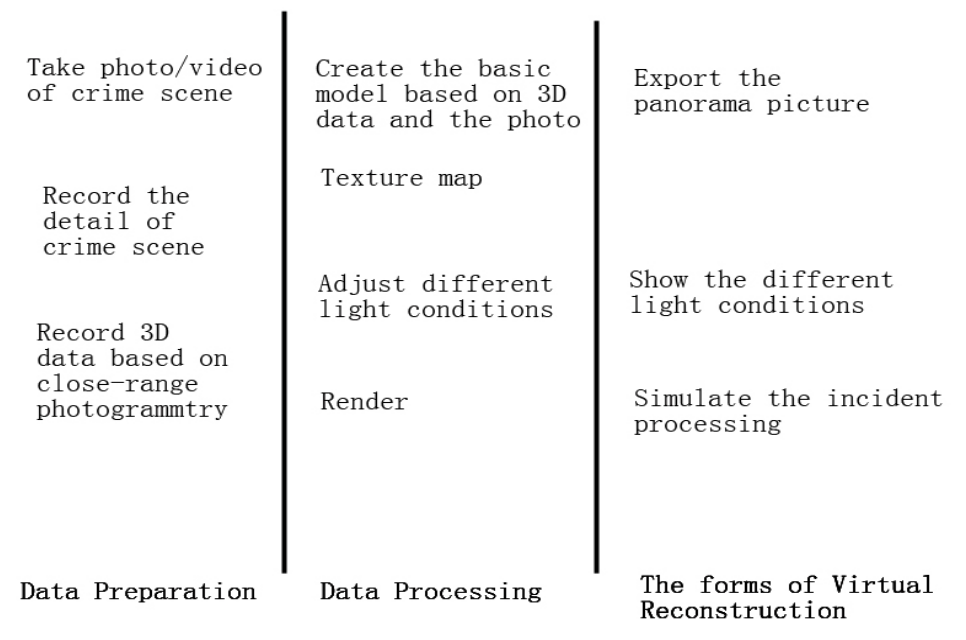

Figure 2: Forensic Visualization Techniques

\subsection{Crime Scene Virtual Tour}

On the basis of 360-degree panoramas, the crime scene virtual tour (CSVT) gives a way to reconstruct a $3 \mathrm{D}$ virtual scene. CSVT takes investigators back to the crime scenes and enables them to wander through the scene.

\section{(1) 3D Reconstruction}

Investigators want to have an overall idea on the place and never lose any suspicious clew. Compared with conventional photograph, the 3D reconstruction of crime scene is not just an album of photographs with 360-degree file of view, but a kind of integration of a variety of scene elements, which brings you back to the actual place where the crime takes place.

\section{(2) Simulation}

As mentioned above, the bullet trajectory could be simulated in the virtual space. CSVT provides a virtual roaming mode; besides, you can simulate the occurrence of crime case by $3 \mathrm{D}$ animation, then link them in the relevant panorama with hotspots. 


\section{(3) Documentation}

Crime Scene Virtual Tour will be more widely used in a forensic science by its great significance during the crime scene investigation.

\section{Case Study}

\subsection{Measure the Position of Corpse}

In the paper, in order to illustrate the state of crime scene, the close-range photogrammetric system and iWitness are used. The non-metric camera is Cannon 600D digital SLR camera with zoom lens EF-S $18-55 \mathrm{~mm}$. We carried the experiment by taken the focal length of $18 \mathrm{~mm}$, aperture F/5.6, ISO 200 and iWitness photogrammetry software.

The procedure is further detailed in iWitness Users Manual. The schematic form the Corpse of the field position is shown in Fig. 3 and the measurement results are shown in Table 1.

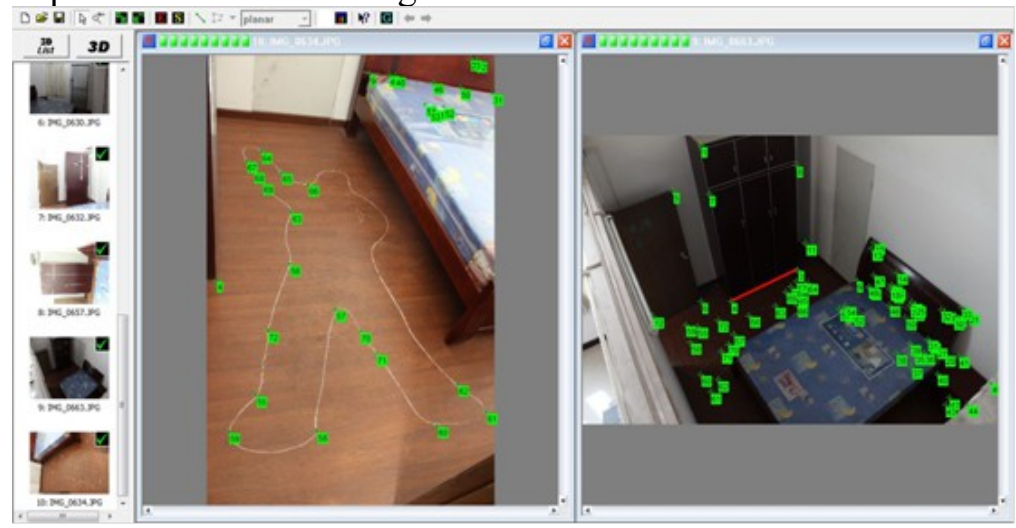

Figure 3: Corpse Status in Crime Scene

\begin{tabular}{|l|l|l|l|}
\hline Distance & $\begin{array}{l}\text { Measured by } \\
\text { Photogrammetry }\end{array}$ & $\begin{array}{l}\text { The actual } \\
\text { distance }\end{array}$ & Errors(\%) \\
\hline Right Knee to East Wall & $200.3 \mathrm{~cm}$ & $201.1 \mathrm{~cm}$ & $0.40 \%$ \\
\hline Right Head to Sofa & $24.7 \mathrm{~cm}$ & $24.9 \mathrm{~cm}$ & $0.80 \%$ \\
\hline Right Foot to North Wall & $227.6 \mathrm{~cm}$ & $227.1 \mathrm{~cm}$ & $0.22 \%$ \\
\hline Head to Door & $202.4 \mathrm{~cm}$ & $200.9 \mathrm{~cm}$ & $0.75 \%$ \\
\hline
\end{tabular}

Table1: Measurement Data of Corpse Location

By means of experiment, compared to the traditional method and error analyzing, it is proved that the principle and methods are available.

\subsection{Virtual Crime Scene}

In order to completely record the crime scene, criminal photography and video will not only take the spot but also take the orientation, circumstances surrounding of crime scene and so on. There are two ways to get panoramas as mentioned in 2.2.1 and 2.2.2. We choose CANNON whole site camera, fisheye lens, use a tripod to capture images through five directions (front, back, right, left and top), the interval is 90 degrees, and then stitch the images.

\subsection{Crime Scene Virtual Tour}

Panorama, as a deformed image, doesn't meet the visual habit. Panoramic roaming, also called the virtual space navigation, is actually a drawing process based on the panorama scene and the representation of the scene model. We choose Pano2VR software for virtual tour of the crime scene. Fig. 4 shows details of Pano2VR. 

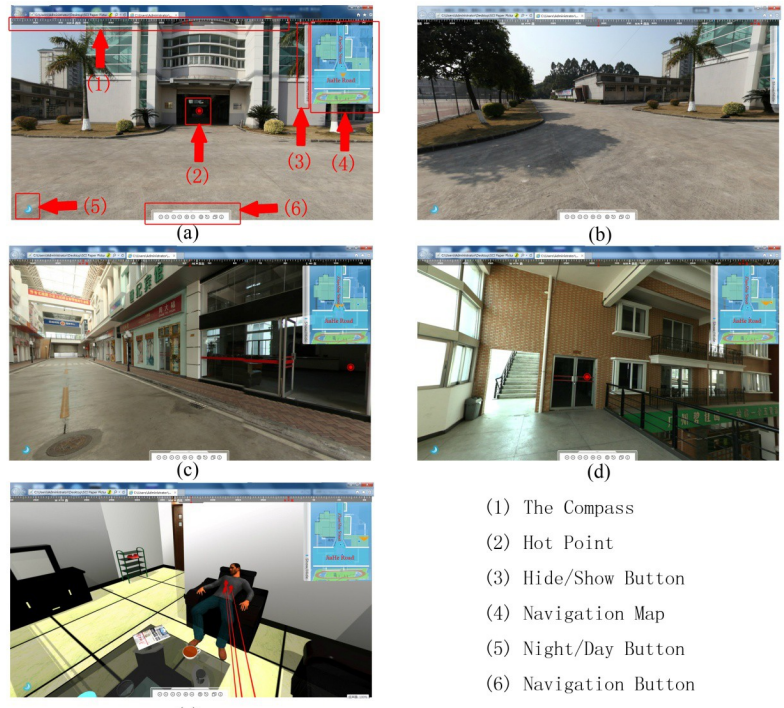

(1) The Compass
(2) Hot Point
(3) Hide/Show Button
(4) Navigation Map
(5) Night/Day Button
(6) Navigation Button

Figure 4: Virtual Tour of Crime Scene at Different Places

\section{Conclusion}

In the paper, based on the detailed and in-depth analysis, the defects of different technologies have been studied and discussed. In combination with the modern technologies and crime scene reconstruction theory, a novel method for virtual crime scene has been present. The main research content and innovative work are providing affordable package solutions for crime site analysis and research, as well as the court cross-examination, including data measurement, live virtual display, simulated conditions analysis, and so on. As there are also some drawbacks, for example, it couldn't support the $3 \mathrm{~d}$ cloud for crime site, several future works will be investigated in detail.

(1) With the development of technology, the price of 3d laser equipment will fall and the department will afford such cost; so how to use the equipment effectively is worth considering.

(2) How to train relevant personnel to promote a virtual reconstruction of the scene is worth considering.

(3) In order to learn how events may occur at the crime scene, the virtual reality provides a new solution for the problem.

(4) The development of virtual environments for police training is a fruitful area for exploration.

\section{References}

[1] EW Durnal. Crime scene investigation (as seen on TV) [J]. Forensic Science International. 199(1-3), 1-5(2010)

[2] TK.Dang, M. Worring, T.D. Bui. A semi-interactive panorama based $3 D$ reconstruction framework for indoor scenes [J]. Computer Vision and Image Understanding. 115(11), 1516-1524 (2011)

[3] P. Delaurentis. 3D scanning: A new tool for cracking tough cases [J]. Forensic Magazine. 6(1): 37-40(2009)

[4] SL Davy-Jow, DM Lees, S Russell S. Viual forensic anthropology: Novel applications of anthropometry and technology in a child death case [J]. Forensic Science International. 224(1-3), 710(2013) 
[5] T Colard, Y Delannoy YF Bresson F, Marechal, JS Raul, V Hedouinet al. SCT imaging of bullet trajectory in $3 D$ crime scene reconstruction: Two case reports [J]. Legal Medicine. 15(6), 318322(2013)

[6] MH Ma, HRuirug, Harjinder Virtual reality and 3D animation in forensic visualization [J]. Journal of Forensic Sciences. 55(5), 1227-1231(2010)

[7] DC Leet, C Gary. Cooperange Photogrammetry for Sightline Obstruction Determination [J]. Evidence Technology Magazine. 10(5), 18-22(2012)

[8] Cl Fraser. Automatic camera calibration in close range photogrammetry [J]. Photogrammetric Engineering and Remote Sensing. 79(4), 381-388(2013)

[9] GJ Liao, H.. u. Study on the crime scene virtual reconstruction based on image rendering [J]. Journal of Chinese People's Public Security University (Science and Technology). 20(79), 2831(2014) (In Chinese)

[10] M Chen, YH. heng, Y.N en, JW Shen, HJ Suet altual geographic environments oriented 3D visualization system [J]. Journal of System Simulation. 20(19), 5105-5108(2008) 